\title{
A FOSSIL BULGE GLOBULAR CLUSTER REVEALED BY VERY LARGE TELESCOPE MULTI-CONJUGATE ADAPTIVE OPTICS*
}

\author{
Sergio Ortolani ${ }^{1}$, Beatriz Barbuy ${ }^{2}$, Yazan Momany $^{3,4}$, Ivo Saviane $^{3}$, Eduardo Bica ${ }^{5}$, \\ LuCIE Jilkova ${ }^{3,6}$, GuStavo M. SALERNO ${ }^{5}$, AND BRUNo JUNGWIERT ${ }^{7,8}$ \\ ${ }^{1}$ Dipartimento di Astronomia, Università di Padova, Vicolo dell'Osservatorio 2, I-35122 Padova, Italy; sergio.ortolani@ unipd.it \\ ${ }^{2}$ Instituto de Astronomia, Geofísica e Ciências Atmosféricas da USP, Rua do Matão 1226, São Paulo 05508-900, Brazil; barbuy @ astro.iag.usp.br \\ ${ }_{3}^{3}$ European Southern Observatory, Casilla 19001, Santiago 19, Chile; ymomany @eso.org, isaviane@eso.org, 1jilkova@eso.org \\ ${ }^{4}$ Osservatorio Astronomico di Padova, Vicolo dell'Osservatorio 5, I-35122, Padova, Italy \\ ${ }^{5}$ Departamento de Astronomia, Universidade Federal do Rio Grande do Sul, CP 15051, Porto Alegre 91501-970, Brazil; bica@if.ufrgs.br, salerno@if.ufrgs.br \\ ${ }^{6}$ Department of Theoretical Physics and Astrophysics, Faculty of Science, Masaryk University, Kotlářská 2, CZ-611 37 Brno, Czech Republic \\ ${ }^{7}$ Astronomical Institute, Academy of Sciences of the Czech Republic, Bo ční II 1401/1a, CZ-141 31 Prague, Czech Republic \\ ${ }^{8}$ Astronomical Institute, Faculty of Mathematics and Physics, Charles University in Prague, Ke Karlovu 3, CZ-121 16 Prague, Czech Republic \\ Received 2010 June 16; accepted 2011 May 25; published 2011 July 26
}

\begin{abstract}
The globular cluster HP 1 is projected on the bulge, very close to the Galactic center. The Multi-Conjugate Adaptive Optics Demonstrator on the Very Large Telescope allowed us to acquire high-resolution deep images that, combined with first epoch New Technology Telescope data, enabled us to derive accurate proper motions. The cluster and bulge fields' stellar contents were disentangled through this process and produced an unprecedented definition in color-magnitude diagrams of this cluster. The metallicity of $[\mathrm{Fe} / \mathrm{H}] \approx-1.0$ from previous spectroscopic analysis is confirmed, which together with an extended blue horizontal branch imply an age older than the halo average. Orbit reconstruction results suggest that HP 1 is spatially confined within the bulge.
\end{abstract}

Key words: Galaxy: bulge - globular clusters: general - globular clusters: individual (HP 1)

Online-only material: color figures

\section{INTRODUCTION}

A deeper understanding of the globular cluster population in the Galactic bulge is becoming possible, thanks to highresolution spectroscopy and deep photometry using $8-10 \mathrm{~m}$ telescopes. An interesting class concerns moderately metal-poor globular clusters $([\mathrm{Fe} / \mathrm{H}] \approx-1.0)$, showing a blue horizontal branch (BHB). A dozen of these objects are found projected on the bulge (Barbuy et al. 2009), and they might represent the oldest globular clusters formed in the Galaxy.

According to Gao et al. (2010), the first generation of massive, fast-evolving stars formed at redshifts as high as $z \approx 35$. Second-generation low-mass stars would be found primarily in the inner parts of the present-day Galaxy as well as inside satellite galaxies. Nakasato \& Nomoto (2003) suggest that the metal-poor component of the Galactic bulge should have formed through a subgalactic clump merger process in the protoGalaxy, where star formation would be induced and chemical enrichment occurred by Type II supernovae. The metal-rich component instead would have formed gradually in the inner disk. Therefore, metal-poor inner-bulge globular clusters might be relics of an early generation of long-lived stars formed in the proto-Galaxy.

Another aspect of interest in inner bulge studies comes from evidence that stellar populations in the Galactic bulge are similar to those in spiral bulges and elliptical galaxies, and therefore are of great interest as templates for the study of external galaxies (Bica 1988; Rich 1988). In the past, the detailed study of the bulge globular clusters was hampered by high reddening and crowding. With the improvement of instrumentation, it is now possible to derive accurate proper motions and to apply

\footnotetext{
* The observations were collected with the Very Large Telescope (Melipal) at the European Southern Observatory, ESO, in Paranal, Chile; the run was carried out in Science Demonstration Time.
}

membership cleaning. Most previous efforts in proper motion studies were carried out using the Hubble Space Telescope (HST) data by our group (e.g., Zoccali et al. 2001) for NGC 6553, Feltzing \& Johnson (2002) for NGC 6528, and Kuijken \& Rich (2002) for field stars, among others.

In a systematic study of bulge globular clusters (e.g., Barbuy et al. 1998, 2009), we identified a sample of moderately metal-poor clusters $([\mathrm{Fe} / \mathrm{H}] \sim-1.0)$ concentrated close to the Galactic center. HP 1 has a relatively low reddening, and appeared to us as a suitable target to be explored in detail in terms of proper motions. Its coordinates are $\alpha=17^{\mathrm{h}} 31^{\mathrm{m}} 05^{\mathrm{s}} .2$, $\delta=-29^{\circ} 58^{\prime} 54^{\prime \prime}(\mathrm{J} 2000)$, and it is projected at only 3.33 from the Galactic center $(l=-2.5748, b=2.1151)$.

In 2008, the European Southern Observatory (ESO) announced a public call for Science Verification of the MultiConjugate Adaptive Optics Demonstrator (MAD; Marchetti et al. 2007), installed at the ESO Melipal (UT3) Telescope. Several star clusters were observed with the MAD facility, delivering high-quality data for the open clusters Trapezium, FSR 1415, and Trumpler 15 (Bouy et al. 2008; Momany et al. 2008; Sana et al. 2010), the globular clusters Terzan 5 and NGC 3201 (Ferraro et al. 2009; Bono et al. 2010), and 30 Doradus (Campbell et al. 2010). The major advantage of MAD is that it allows correcting for atmospheric turbulence over a wide $2^{\prime}$ diameter field of view, and as such constitutes a pathfinder experiment for future facilities at the European Extremely Large Telescope (E-ELT). Wide-field adaptive optics with large telescopes open a new frontier in determining accurate parameters for most globular clusters that remain essentially unstudied because of high reddening, crowding (cluster and/or field), and large distances.

In Section 2 the observations and data reductions are described. In Section 3 the HP 1 proper motions are derived. The impact of the high-quality proper motion-cleaned 


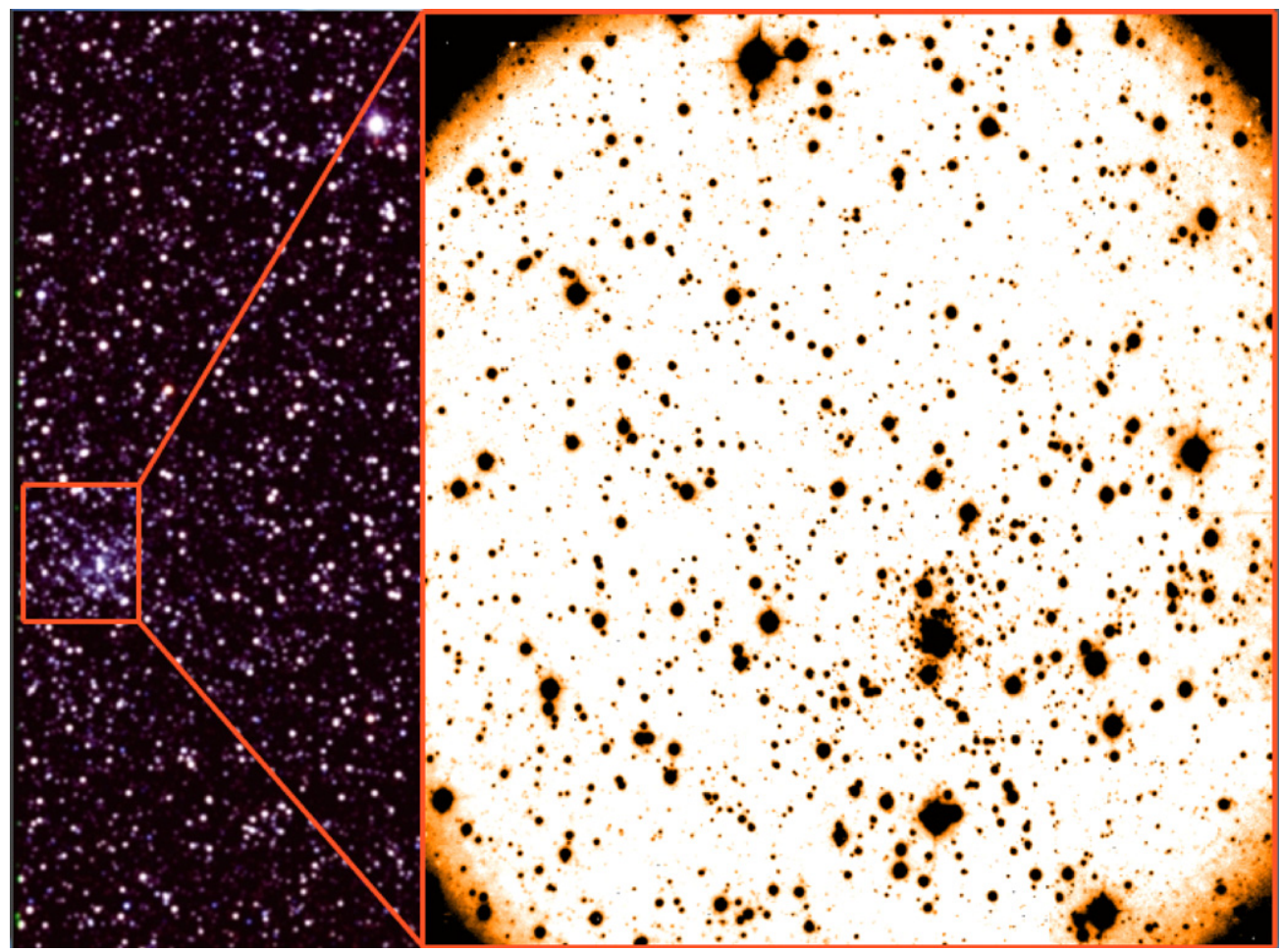

Figure 1. MAD image of the globular cluster HP 1, obtained at ESO-VLT at Paranal, Chile. The left panel is a composite color image of HP 1 from the near-infrared 2MASS survey. The right panel is a close-up of the MAD $K$-band image covering the central 1 $1.8 \times 11^{\prime} 8$ of HP 1 . North is up and east is to the left.

(A color version of this figure is available in the online journal.)

Table 1

Log of the MAD Observations Obtained on 2008 August 15

\begin{tabular}{lcccc}
\hline \hline Filter & FWHM & Airmass & DIT (s) & NDIT \\
\hline$K$ & $0 ! .19$ & 1.149 & 10 & 30 \\
$K$ & $0 ! 21$ & 1.179 & 10 & 30 \\
$K$ & $0 ! 21$ & 1.213 & 10 & 30 \\
$K$ & $0 ! 27$ & 1.260 & 10 & 30 \\
$K$ & $0 ! 26$ & 1.304 & 10 & 30 \\
\hline$J$ & $0 ! ' 35$ & 1.081 & 10 & 30 \\
$J$ & $0 ! 41$ & 1.101 & 10 & 30 \\
$J$ & $0 ! 32$ & 1.124 & 10 & 30 \\
$J$ & $0 ! 33$ & 1.152 & 10 & 30 \\
$J$ & $0 ! 42$ & 1.183 & 10 & 30 \\
\hline
\end{tabular}

color-magnitude diagrams (CMDs) on cluster properties is examined in Section 4. The clusters' orbit in the Galaxy is reconstructed in Section 5. Finally, conclusions are given in Section 6.

\section{OBSERVATIONS AND DATA ANALYSIS}

MAD was developed by the ESO Adaptive Optics Department to be used as a visitor instrument on the Melipal Unit Telescope 3 at the Very Large Telescope (VLT) with a view toward future application to the E-ELT. It was installed at the visitor Nasmyth focus, and the concept of multiple reference stars for layer-oriented adaptive optics corrections was introduced. This allows a much wider and more uniform corrected field of view, providing larger average Strehl ratios and making the system a powerful diffraction limit imager. This is particularly important in crowded fields where photometric accuracy is needed. ${ }^{9}$ Following our successful use of MAD in the first

\footnotetext{
http://www.eso.org/projects/aot/mad/
}

Science Demonstration (Momany et al. 2008), we were granted time to observe HP 1 in the second Science Demonstration. ${ }^{10}$

HP 1 was observed on 2008 August 15. Table 1 displays the $\log$ of the $J$ and $K_{\mathrm{s}}$ (for brevity we use $K$ ) observations. Clearly, the seeing in $K$ was excellent, being almost half that in $J(0.23$ versus $\left.0{ }^{\prime} .38\right)$. The MAD infrared scientific imaging camera is based on a $2048 \times 2048$ pixel HAWAII- 2 infrared detector with a pixel scale of 0.028 . In total, 25 minutes of scientific exposures were dedicated to each filter, and subdivided into five dithered images.

The images were dark and sky-subtracted, and then flatfielded following the standard near-infrared recipes (e.g., Momany et al. 2003), within the IRAF environment. The typical dithering pattern of a MAD observation allows a field of view of $\sim 2^{\prime}$ in diameter. Within this field of view, three reference bright stars ( $R$ magnitudes that ranged between 12.5 and 13.8 according to their magnitudes in the second US Naval Observatory CCD Astrograph Catalog (UCAC2)) were selected to ensure the optics correction. However, one of these proved to be a blend of two stars, which did not allow a full optical correction of the field.

Figure 1 shows the mosaic of all $10 \mathrm{~J}$ and $K$ images as constructed by the DAOPHOT/MONTAGE2 task. The superb VLT/MAD resolution is illustrated when compared to that seen in the Two Micron All Sky Survey (2MASS) $K$ image of HP 1. For proper motion purposes, the MAD data set represents our second epoch data. The displacement of the HP 1 stellar content was derived by comparing the position of the stars in this data set with respect to that obtained with the SUSI CCD camera at the New Technology Telescope (NTT) on 1994 May 16 (Ortolani et al. 1997). The epoch separation is $14.25 \mathrm{yr}$. It is

\footnotetext{
10 http://www.eso.org/sci/activities/vltsv/mad/
} 
worth emphasizing that the seeing of 0.45 for the first epoch $\mathrm{V}$ image of HP 1 is one of the best obtained with NTT (Ortolani et al. 1995, 1997).

The photometric reductions of the two data sets (NTT and MAD) were carried out separately. We found about 3100 stars in common between the MAD and NTT data, which were used for proper motion analyses.

\subsection{Photometric Reduction and Calibration}

The stellar photometry and astrometry were obtained by point-spread function (PSF) fitting using DAOPHOT II/ ALLFRAME (Stetson 1994). Once the FIND and PHOT tasks were performed and the stellar-like sources were detected, we searched for isolated stars to build the PSF for each single image. The final PSF was generated with a PENNY function that had a quadratic dependence on frame position. ALLFRAME combines PSF photometry carried out on the individual images and allows the creation of a master list by combining images from different filters. This pushes the detection limit to fainter magnitudes and provides better determination of the stellar magnitudes (given that five measurements were used for each detected star).

Our observing strategy employed the same exposure time for all images, and no bright red giant stars were saturated. When producing the photometric catalog in one filter (and since only the central part of the field of view had multiple measurements of any star), stars appearing in any single image were considered to be real. Later, when producing the final $J K$ color catalog, only those appearing in both filters were recorded (this way we essentially removed all detections due to cosmic rays and other spurious detections). The photometric catalog was finally transformed into coordinates with astrometric precision by using 12 UCAC2 reference $\operatorname{stars}^{11}$ with $R \leqslant 16.2$.

Photometric calibration of the $J$ and $K$ data has been made possible by a direct comparison of the brightest MAD nonsaturated $(8.0 \leqslant K \leqslant 13.0)$ stars with their 2MASS counterpart photometry. From these stars we estimate a mean offset of $\Delta J_{\mathrm{J} @ \mathrm{MAD}-\mathrm{J} @ 2 \mathrm{MASS}}=-3.157 \pm 0.120$ and $\Delta K_{\mathrm{K} @ \mathrm{MAD}-\mathrm{K} @ 2 \mathrm{MASS}}=-1.395 \pm 0.134$.

Photometric errors and completeness were estimated from artificial star experiments previously applied to similar MAD data (Momany et al. 2008). The images with added artificial stars were reprocessed in the same manner as the original images. The results for photometric completeness showed that we reach a photometric completeness of $\sim 75 \%, 50 \%$, and $10 \%$ around $K \simeq 17.0,17.5$, and $\sim 18.0$, respectively.

\section{DERIVATION OF THE PROPER MOTIONS}

The proper motion of the HP 1 stellar content was derived by estimating the displacement in the $(x, y)$ instrumental coordinates between the MAD (second epoch) data and the NTT (first epoch) data. Since this measurement was made with respect to reference stars that are cluster members, the motion zero point is the centroid motion of the cluster. The small MAD field of view is fully sampled by the wider NTT coverage, and thus essentially all MAD entries had NTT counterparts for proper motion determination. In this regard, we note that the photometric completeness of the MAD data set is less than that of the optical $V, I$ NTT data that reached at least two magnitudes below the cluster turnoff (Ortolani et al. 1997).

\footnotetext{
11 http://www.usno.navy.mil/USNO/astrometry/optical-IR-prod/ucac
}

The DAOPHOT/DAOMASTER task was used to match the photometric MAD and NTT catalogs, using their respective $(x, y)$ instrumental coordinates. This task employed a cubic transformation function, which, with a matching radius of 0.5 pixels or 0.015 , easily identified reference stars among the cluster's stars (having similar proper motions). As a consequence, the stars that matched between the two catalogs were basically only HP 1 stars. In a separate procedure, the J2000 MAD and NTT catalogs, transformed into astrometric coordinates, were applied to a matching procedure using the IRAF/TMATCH task. This first/second epoch merged file also included the $(x, y)$ instrumental coordinates of each catalog. Thus, applying cubic transformation to both coordinate systems yielded the displacement with respect to the centroid motion of HP 1 . An extraction within a 1.5 pixel radius around $(0,0)$ in pixel displacement was shown to contain most, and essentially only, HP 1 stars, and we will use this selection for the rest of the paper.

On the other hand, stars outside this radius (i.e., representing the bulge populations) are more dispersed and required a careful analysis. In a first attempt, we used only the stars with $\Delta y<-1$ (in order to avoid cluster stars) to get the barycentric position of the field bulge population in $x$, and stars with $\Delta x>1$ to measure the field bulge population in $y$. After a number of tests with different selections, in a second attempt we made a twoGaussian component fit along a 0.2 pixel-wide strip connecting the center of the cluster distribution and the field distribution. For the present analysis, we adopted relative to the field the mean of the two determinations $(\Delta x, \Delta y)=(-2.1,1.96)$ for the proper motion of the cluster. From the Gaussian fit we derived the width of the distributions of the cluster and field stars, resulting, respectively, in $\sigma=0.583$ and 2.565 pixels, corresponding to 15 and 41 mas, respectively. This is the error of the single star position. The statistical error of the mean is obtained by dividing the single star error by the square root of the number of stars used (about 1700 in the cluster and 1500 in the bulge) for each of the two measurements. The error in the mean position of the field obviously dominates and corresponds to about 0.05

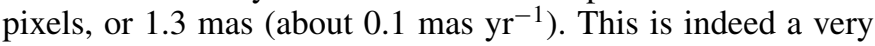
small error. We performed a number of tests fitting the cluster stars and the field stars in different conditions checking for additional uncertainties. A further quantitative evaluation of the fitting uncertainty can be obtained by measuring the different distances between the centers of the HP 1 proper motion cloud to that of the field, propagated from the error on the angle of the line connecting the two groups of stars. The angle of the vector joining the center of the HP 1 proper motion cloud to that of each star of the bulge was computed, and a Gaussian fit was performed, which returned a $1 \sigma$ dispersion of $14^{\circ}$. This uncertainty propagates over the distances of the two groups by about 0.39 mas $\mathrm{yr}^{-1}$, dominating over the other discussed sources.

\subsection{Astrometric Errors}

The astrometric errors are the combination of different random and systematic errors. Anderson et al. (2006) concluded that in the case of relative ground-based astrometry in a small rich field, the main error sources are random centering errors due to noise and blending, random-systematic errors due to field distortions, and systematic errors due to chromatic effects.

1. Centering errors. Considering that we used two very different data sets-a first epoch based on direct CCD photometry, and a second one on the corrected infrared adaptive 
optics (with a much wider scale and better resolution) — we can assume that the astrometric random errors are dominated by the first epoch classical CCD photometry. Following Anderson et al. (2006), we performed a test with three consecutive NTT images of Baade's Window taken in the same night. They were obtained with equal (four-minute) exposure times, and nearly at the same pixel positions. The seeing was stable and these images were obtained about $1 \mathrm{hr}$ later than the 10 minute cluster $V$ exposure.

This Baade's Window field was chosen because the stars are uniformly distributed across the image, and the density of stars is similar to the HP 1 field. These images were analyzed in Ortolani et al. (1995). The position of the stars in common was compared. The photometric and astrometric errors are shown in Figure 2. The astrometric centering error, averaged over the whole dynamic range, is 0.1 pixels, as indicated in Figure 2. This corresponds to 13 mas, which is comparable with the 7 mas pointing error by Anderson et al. (2006). The value of 13 mas amounts to $0.91{\text { mas } \mathrm{yr}^{-1}}^{-1}$ in an interval of $14.25 \mathrm{yr}$. Taking into account the number of about 3000 stars (half field and half cluster) used for the proper motion derivation of HP 1, this leads to a minor error of 0.022 mas.

2. Field distortion errors. The field distortion analysis is usually performed employing shifted images of a field with uniformly distributed stars. HP 1 multiple shifted images are not available in our first epoch run. To estimate the distortions, we compared the proper motion of cluster stars in HP 1 NTT images as a function of the distance from the optical center. The distribution did not show any relevant trend, and the statistical analysis indicates that there is no significant shift above 0.01 pixels across the field. This

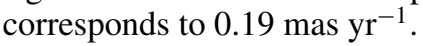

3. Chromatic errors. Chromatic errors are due to the different refraction, both atmospheric and instrumental, on stars with different colors. The refraction dependence on wavelength (inverse quadratic to the first approximation) makes this effect more pronounced in the color $(V, I)$ than in the nearinfrared.

Ideally, a chromatic experiment should include a measurement of the displacement of stars with different colors at different air masses. However, we do not have specific observations for this test. Thus, we followed the procedure given by Anderson et al. (2006), where they measured the displacement of stars as a function of their colors. We took the resulting pixel displacement of the cluster stars and separately checked the shift variations with the color $(V-I)$. Such a plot does not show an evident dependence on color. This is expected since our NTT observations were taken very close to the zenith (air mass $\sim 1.04$ ), and the infrared MAD data have a negligible effect.

In order to further quantify any chromatic systematic effect, we subdivided the sample of cluster stars into redder $(V-I>2.2)$ and bluer $(V-I<2.2)$ groups, of 106 and 171 stars, respectively. The displacement between the two groups resulted is $0.031 \pm 0.03$ pixels, corresponding to

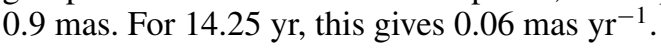

4. Total errors. By quadratically adding the errors on centering, distortion, and chromatic effects of, respectively,

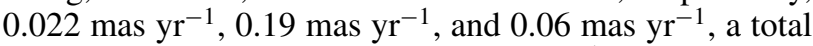

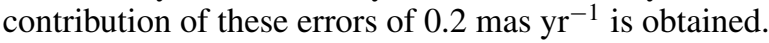

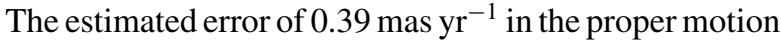
value indicates that the effect due to the mutual field and
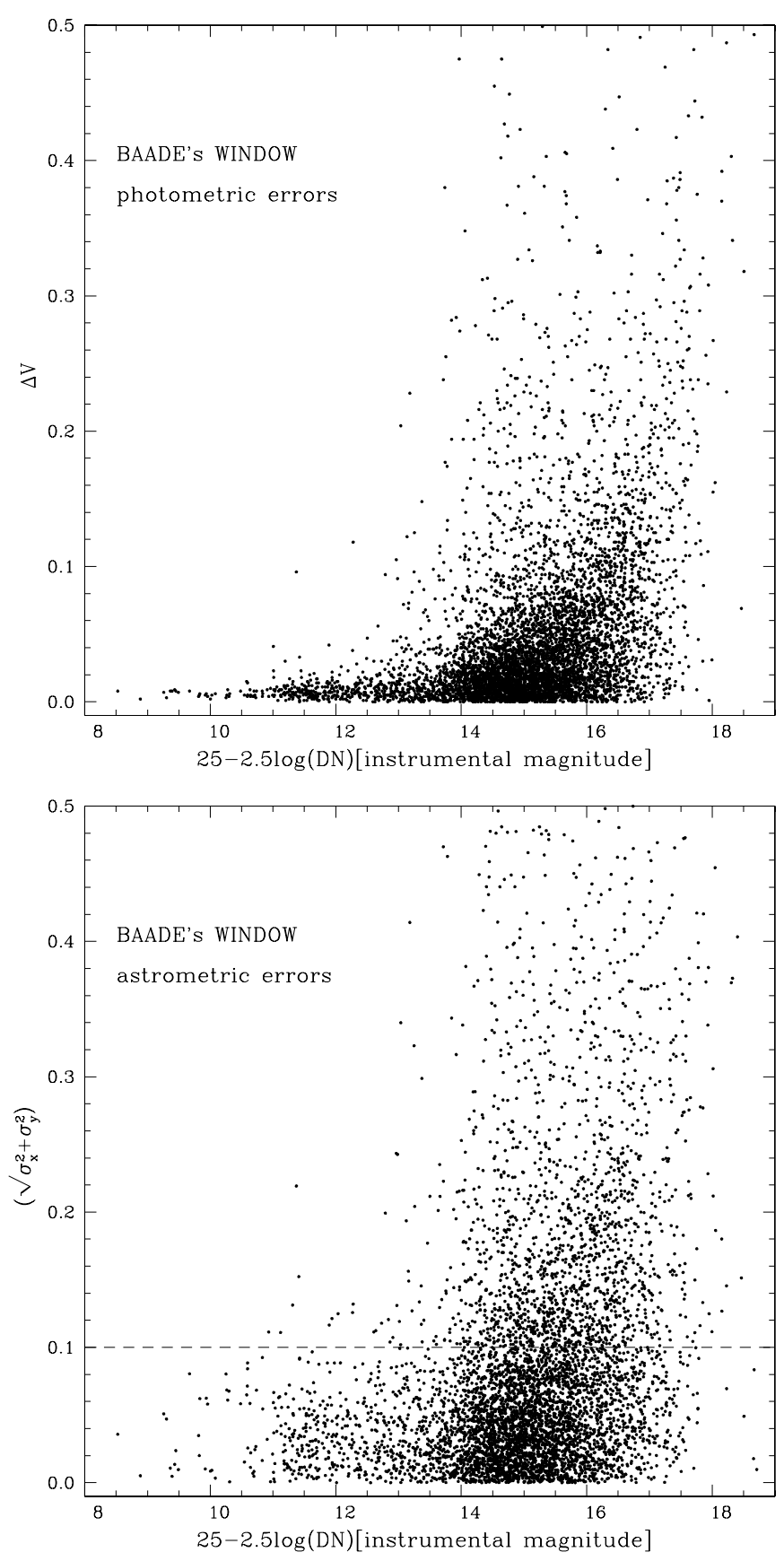

Figure 2. Photometric and astrometric errors of Baade's Window images. Top panel: photometric errors $\Delta V$ vs. instrumental magnitude. The dashed line shows the average astrometric error. Bottom panel: astrometric error in the NTT pixel scale vs. instrumental magnitude.

cluster star contamination dominates over the astrometric pointing, distortion, and chromatic errors.

\section{THE PROPER MOTION-CLEANED COLOR-MAGNITUDE DIAGRAM OF HP 1}

Figure 3 presents the MAD $K$ versus $J-K$ CMDs showing the proper motion decontamination process in three panels: the whole field, the cluster proper motion decontaminated, and the remaining field only. In the top panels the displacements of stars between the NTT and MAD images are shown, plotted in the MAD pixel scale. 


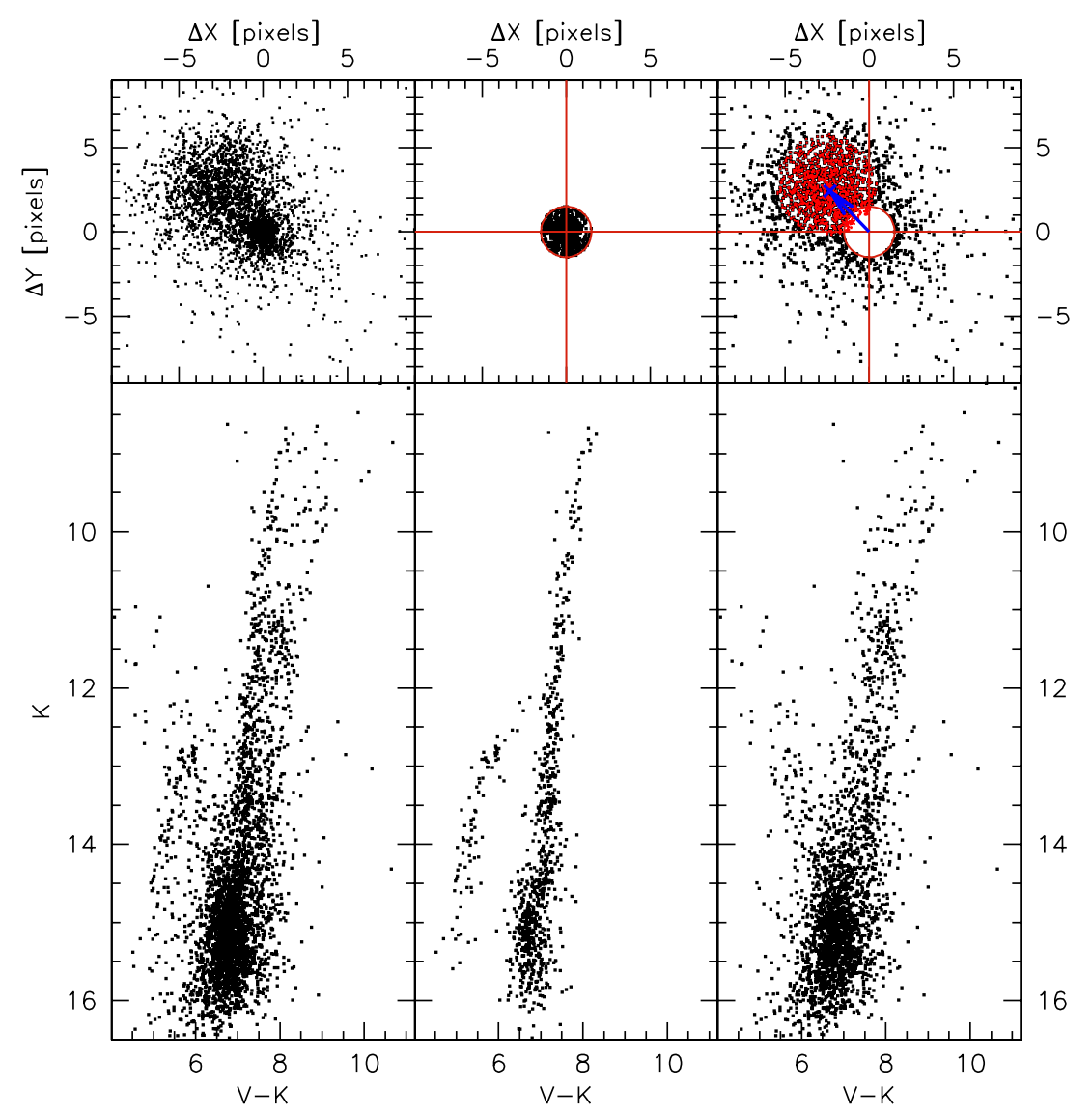

Figure 3. HP 1: proper motion decontaminated $K$ vs. $J-K$ CMD. Top left panel: displacement of NTT (first epoch)-MAD (second epoch), plotted in the MAD pixel scale. Top middle panel: the cluster sample is encircled and $l, b$ directions are indicated by the arrows. Top right panel: field sample only. Bottom left panel: observed CMD. Bottom middle panel: proper motion decontaminated cluster CMD. The two stars indicated as red squares are the two giant stars analyzed with high-resolution spectroscopy. Bottom right panel: remaining field star CMD. The extraction is within a 1.5 pixel radius.

(A color version of this figure is available in the online journal.)

The decontaminated cluster CMD provides fundamental parameters for studying its properties, in particular metallicity and age. Figure 4 shows a $J$ versus $V-K$ proper motion decontaminated CMD of HP 1. A distance modulus of $(m-M)_{\mathrm{J}}=15.3$ and a reddening of $E(V-K)=3.3$ are applied, and a Padova isochrone (Marigo et al. 2008) with metallicity $Z=0.002$ and an age of $13.7 \mathrm{Gyr}$ is overplotted. The fit confirms the cluster metallicity of $[\mathrm{Fe} / \mathrm{H}]=-1.0$, found from highresolution spectroscopy (Barbuy et al. 2006). We note that the turnoff is not as well matched as the giant branch, due to two well known main reasons: the bluer turnoff in isochrones relative to observations is possibly connected with color transformations, and systematic bias in the photometric errors gives brighter magnitudes close to the limit of the photometry. Similarly, Figure 5 displays the $K$ versus $V-K$ HP 1 diagram as compared with the NGC $6752([\mathrm{Fe} / \mathrm{H}]=-1.42)$ mean locus (Valenti et al. 2004). The red giant branches of M30, M107, 47 Tuc, and NGC 6441 of metallicities $[\mathrm{Fe} / \mathrm{H}]=-1.91,-0.87,-0.70$, and -0.68 , respectively, are also overplotted with metallicity values, mean loci, and red giant fiducials from Valenti et al. (2004). The HP 1 bright red giants clearly overlap with the M107 fiducial (reflecting their similar metallicity values) and are redder with respect to the NGC 6752 bright giants. On the other hand, the cluster's old age is reflected by the presence of a well defined and extended BHB (very similar to NGC 6752). Five RR Lyrae candidates appear in the RR Lyrae gap, at $4.2 \leqslant(V-K) \leqslant 5.0$.
Terzan (1964a, 1964b, 1965, 1966) reported 15 variable stars in HP 1 but none has been identified as RR Lyrae. The Horizontal Branch (HB) morphology is sensitive mainly to metallicity and age. The age effect is related to the so-called second parameter effect (Sandage \& Wildey 1967), also demonstrated in models by Lee et al. (1994), Rey et al. (2001), and in observations by Dotter et al. (2010). In particular, Dotter et al. (2010) analyzed the HB morphology from Advanced Camera for Surveys/HST observations, based on the difference between the average HB $V-I$ color and the subgiant branch (SGB) color $\Delta V-I_{\mathrm{HB}}^{\mathrm{SGB}}$, and concluded that age dominates the second parameter. This indicator is very sensitive to the cluster age, and more so around the metallicity $Z=0.002$.

For HP 1 the mean $V-I$ color difference between the $\mathrm{SGB}$ and the $\mathrm{HB}$ is $\Delta V-I_{\mathrm{HB}}^{\mathrm{SGB}}=0.75 \pm 0.01$. A comparison with Dotter et al.'s (2010) sample shows that HP 1 has a very blue HB for its metallicity. We selected five clusters with the same $\mathrm{HB}$ morphological index and found an average metallicity of $[\mathrm{Fe} / \mathrm{H}]=-1.9 \pm 0.36$. We also selected another group of five clusters with a comparable metallicity to HP 1 . This second group has an average $\Delta V-I=0.46 \pm 0.27$, which is considerably smaller than in HP 1 , consistent with HP 1 having a much bluer HB. Both cluster groups have a mean age of $12.7 \pm 0.4$ Gyr. From Figure 17 of Dotter et al. (2010), we get an age difference of about $1 \mathrm{Gyr}$ older for HP 1 relative to their sample of halo clusters with $\sim 12.7 \mathrm{Gyr}$, resulting in an age of 


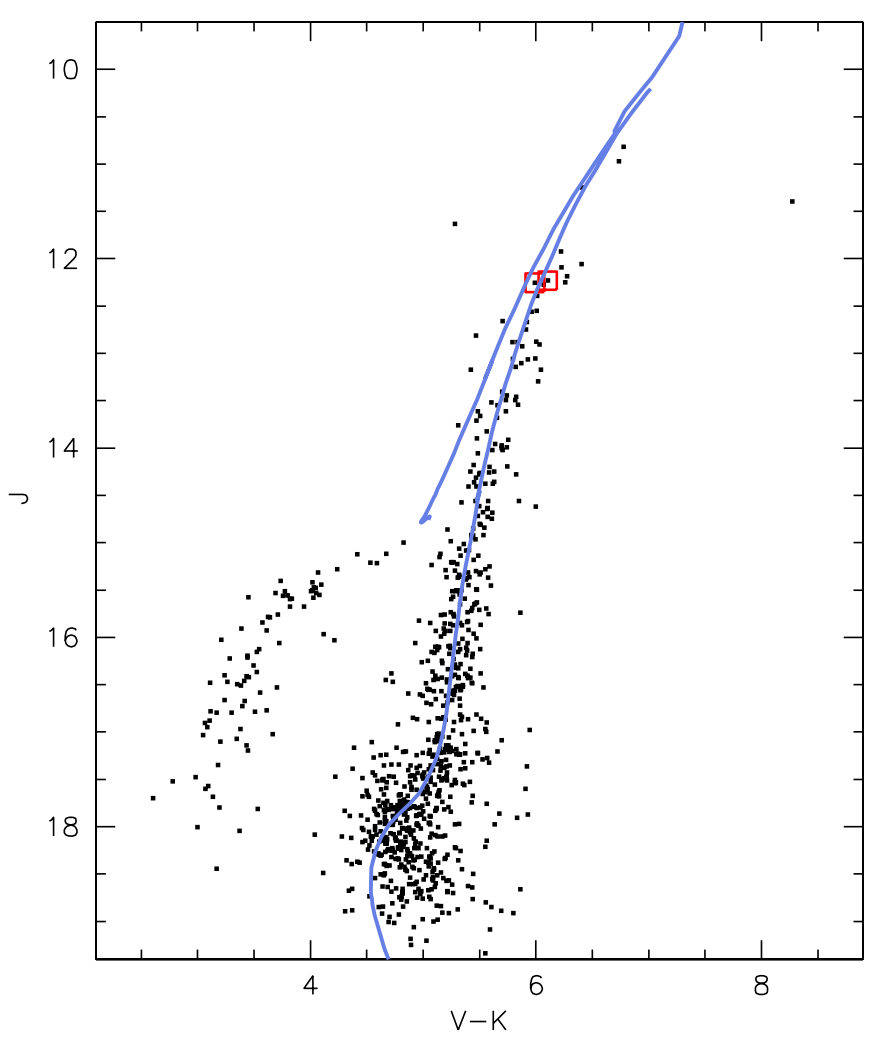

Figure 4. HP 1: $J$ vs. $V-K C \mathrm{CMD}$, built from a proper motion selected subsample of stars. The extraction is within a 1.5 pixel radius, in pixel displacement. A Padova isochrone of $Z=0.002$ and an age of $13.7 \mathrm{Gyr}$ are overplotted on the observed CMD. A distance modulus of $(m-M)_{\mathrm{J}}=15.3$ and a reddening of $E(V-K)=3.3$ were adopted. The metallicity, distance, and reddening adopted for the fit are in agreement with the spectroscopic analysis carried out for two stars indicated by squares (Barbuy et al. 2006). The present MAD photometry reaches the turnoff limit.

(A color version of this figure is available in the online journal.)

13.7 Gyr for HP 1. Therefore, HP 1 appears to be among the oldest globular clusters in the Galaxy.

For the distance determination, there are basically two methods: (1) the relative distance between the cluster and bulk of the bulge field, and (2) based on the absolute distance, which requires reddening values. For the first of these methods, we rely on the difference between the HP $1 \mathrm{HB}$ at the RR Lyrae level, at $V=18.7$, and that of the bulge field at $V=19.35$. Thus, the cluster is $\Delta V=0.66$ brighter than the field, and taking into account metallicity effects on the HB luminosity (Buonanno et al. 1989), we obtain $\Delta V=0.35 \pm 0.14$. This implies that the cluster is $1.2 \pm 0.4 \mathrm{kpc}$ in the foreground of the bulge bulk population. The uncertainty is due to the metallicity difference of about 1 dex between the bulge $([\mathrm{Fe} / \mathrm{H}] \approx 0.0)$ and $\mathrm{HP} 1$ $([\mathrm{Fe} / \mathrm{H}] \approx-1.0)$. Assuming the distance of the Galactic center to be $R_{\mathrm{GC}}=8.0 \pm 0.6$ (Majaess et al. 2009; Vanhollebeke et al. 2009), a distance of $d_{\odot}=6.8 \mathrm{kpc}$ is obtained. If the orbits of stars near the super massive black hole near the Galactic center are used, a distance of $R_{\mathrm{GC}}=8.33 \pm 0.35 \mathrm{kpc}$ is given by Gillessen et al. (2009). In this case the distance of HP 1 to the Sun is $d_{\odot}=7.1 \mathrm{kpc}$. This relative distance method is reddening-independent, because the reddening of the cluster and surrounding field is expected to be essentially the same, due to a negligible reddening inside the bulge (Barbuy et al. 1998). Therefore, the distance of the cluster to the Galactic center depends only on the assumed distance of the Galactic center.

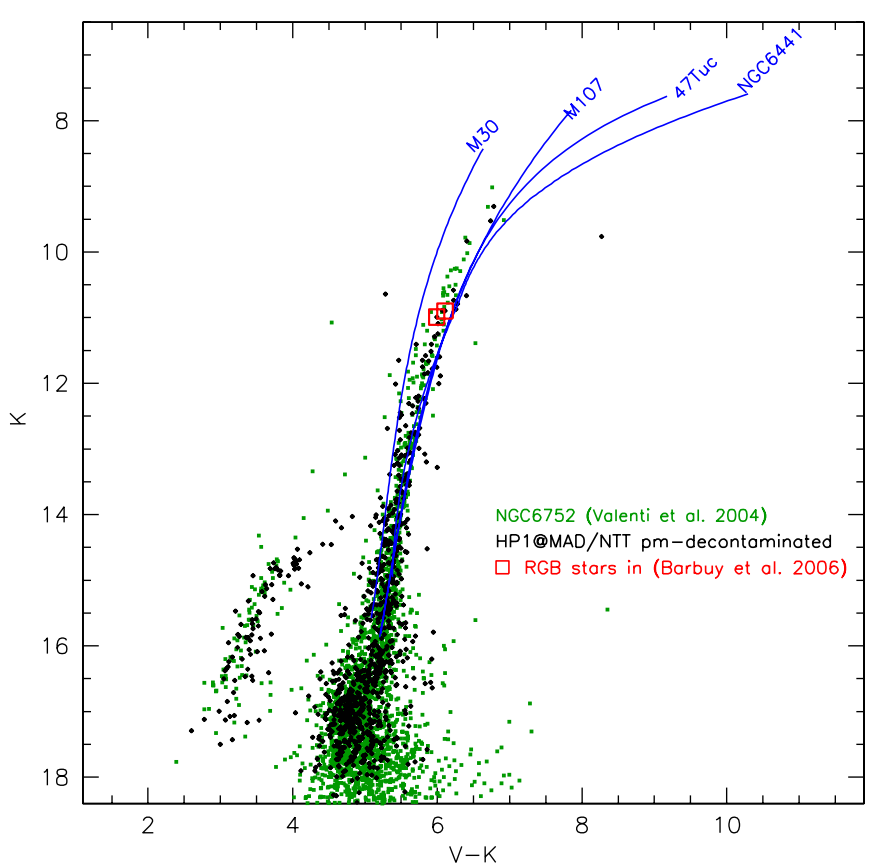

Figure 5. $K$ vs. $V-K$ HP 1 diagram (black dots) compared with the NGC 6752 (green dots) catalog (Valenti et al. 2004). We assumed the fiducial values $(m-M)_{0}=13.18, E(B-V)=0.04, A_{V}=3.1, A_{K}=0.38$. Also plotted are the red giant branches of comparison Galactic globular clusters (blue lines): M30, M107, 47 Tuc, and NGC $6441([\mathrm{Fe} / \mathrm{H}]-2.12,-1.04,-0.76$, and -0.68 ; the catalog and the red giant fiducials are from Valenti et al. 2004). The HP 1 bright red giants are clearly overlapping with the M107 fiducial (reflecting their similar metallicity) and are redder with respect to the NGC 6752 bright giants.

(A color version of this figure is available in the online journal.)

The second method of absolute distances requires reddening determinations. From the optical and infrared CMDs, and adopting the absolute-to-selective absorption $R_{\mathrm{V}}=3.2$ (Barbuy et al. 1998), we obtain a mean distance from the Sun of $d_{\odot}=7.3 \pm 0.5 \mathrm{kpc}$. Within the uncertainties for the cluster and Galactic center distances, we conclude that HP 1 is probably the globular cluster located closest to the Galactic center.

For simplicity, we assume the distance of HP 1 from the Sun to be $d_{\odot}=6.8 \mathrm{kpc}$ hereafter.

\section{SPATIAL MOTION OF HP 1 IN THE GALAXY}

\subsection{Absolute Proper Motion}

To compute the velocity components of HP 1's motion, we need its radial velocity and the proper motion. The heliocentric radial velocity $v_{\mathrm{r}}^{\text {hel. }}=45.8 \pm 0.7 \mathrm{~km} \mathrm{~s}^{-1}$ was adopted from the high-resolution analysis by Barbuy et al. (2006). The proper motion can be computed with respect to the bulge, and the bulge proper motion can then be subtracted. The bulge proper motion is a composition of the bulge internal kinematics and the reflected motion of the LSR. Near the position of HP 1, the bulge kinematics are close to that of a rotating solid body with a spin axis orthogonal to the Galactic plane (e.g., Zhao 1996). Note also that this correction is not large: HP 1 is projected so close to the Galactic center that the rotational velocity of the bulge is closely aligned with the line of sight, so its tangential component is very small. Because both motions are parallel to the Galactic plane, it is convenient to work in Galactic coordinates. 


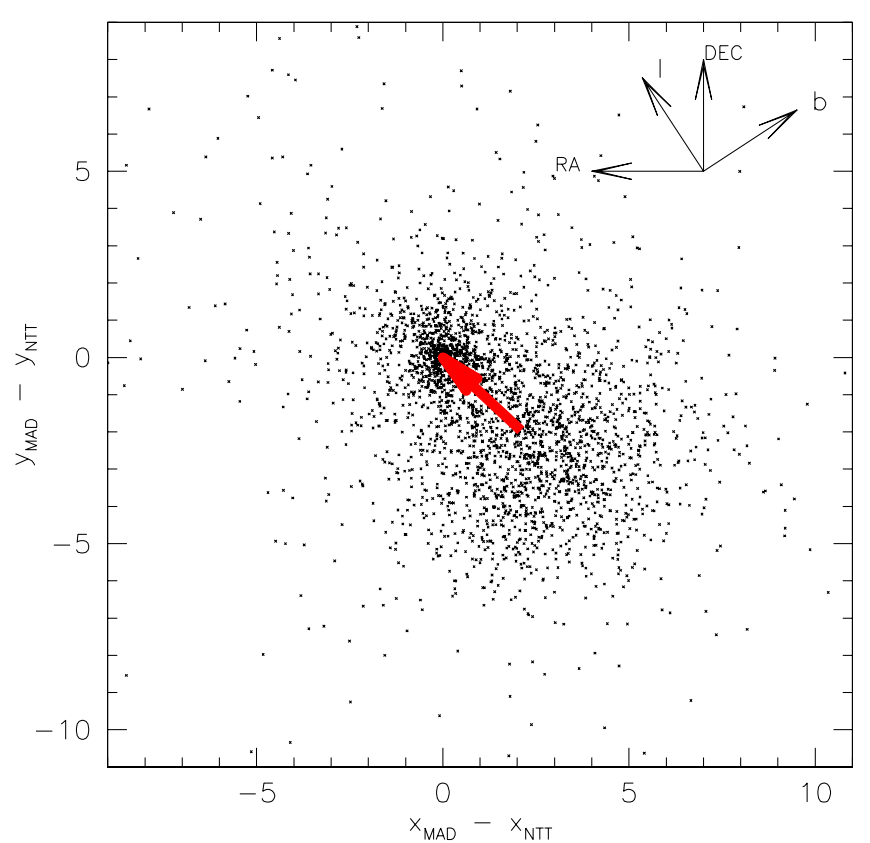

Figure 6. In the reference system $(\Delta x, \Delta y)=\left(x_{\mathrm{MAD}}-x_{\mathrm{NTT}}, y_{\mathrm{MAD}}-y_{\mathrm{NTT}}\right)$ with origin in HP 1 the bulge has the coordinates $(2.10,-1.96)$. Therefore, the HP 1 motion with respect to the bulge has the vector $\overrightarrow{u_{\mathrm{px}}}=(-2.10,1.96)$ (red arrow). Because R.A. and $\Delta x$ have opposite directions, the vector expressed in equatorial coordinates is $\overrightarrow{u_{\mathrm{Eq}}}=\left(0^{\prime \prime} 0588,00^{\prime \prime} 05488\right)$. The relative orientation of the equatorial and Galactic coordinate systems is shown in the upper right corner, making clear that the proper motion of HP 1 is mostly along positive $l$, with a small component along negative $b$. The orbit must therefore be confined near the Galactic plane.

(A color version of this figure is available in the online journal.)

As Figure 6 shows, the HP 1 motion with respect to the bulge has a vector $\overrightarrow{u_{\mathrm{px}}}=(-2.10,1.96)$ pixels. $^{12}$

To estimate the bulge internal motion, we note that Tiede \& Terndrup (1999) obtained a rotational velocity of $\sim 75 \mathrm{~km} \mathrm{~s}^{-1}$ in their fields $6 / 05$ and $6 / 18\left(b=-6^{\circ}\right.$ and the respective field numbers), at positions $l=-8.7$ and $l=8.4$, respectively. In the solid body approximation, $v_{\text {rot }}$ depends linearly on the radius $r$ (confirmed by Howard et al. 2008), and at the position of HP 1 (Section 1) we expect $v_{\text {rot }} \simeq 75 \mathrm{~km} \mathrm{~s}^{-1} / 8.5 \times 2.58=$ $22.8 \mathrm{~km} \mathrm{~s}^{-1}$ (where we assumed $l \propto r$ for small values of $l$ ). Basically the same value $\left(22.7 \mathrm{~km} \mathrm{~s}^{-1}\right)$ is predicted at a distance of $0.38 \mathrm{kpc}\left(=r_{\odot} \times \sin 2.58\right.$ with $\left.r_{\odot}=8.4 \mathrm{kpc}\right)$ by the angular velocity of $60.0 \mathrm{~km} \mathrm{~s}^{-1} \mathrm{kpc}^{-1}$ adopted in our model (Section 5.2). Assuming that bulge stars are at the distance of the Galactic center, the tangential component of $v_{\text {rot }}$ is $v_{\mathrm{T}}=22.8 \mathrm{~km} \mathrm{~s}^{-1} \times \sin 2.58 / \cos 2.58=1.03 \mathrm{~km} \mathrm{~s}^{-1}$

\footnotetext{
12 Note that in Section 3 we were working with NTT-MAD coordinates, while here we put the two epochs in chronological order, thus MAD-NTT coordinates are used. The signs are therefore inverted. Taking into account the scale of $0 . \prime 028$ pixel $^{-1}$ and that $\Delta$ R.A. $=-\Delta x$, the vector expressed in equatorial coordinates is $\overrightarrow{u_{\mathrm{Eq}}}=\left(0^{\prime \prime} 0588,0^{\prime \prime} .05488\right)$. The position angle of the $b$-axis with respect to the decl. axis is -56.745 , which can be used to rotate $\overrightarrow{u_{\mathrm{Eq}}}$, obtaining $\overrightarrow{u_{\mathrm{Gal}}}=(\Delta l, \Delta b)$ with $\Delta l=0^{\prime \prime} 0781 \pm 0$. .'0056 and $\Delta b=-0 . ' 0191 \pm 0$.'0056. The coordinate transformations were performed with the code SM (Lupton \& Monger 1997) using the project package, written by M. Strauss and R. Lupton. With an epoch difference of $\Delta t=14.2519 \pm 0.0027 \mathrm{yr}$, these displacements yield proper motion components relative to the bulge of $\mu_{l}^{\text {rel }} \times \cos b \simeq 5.5 \mathrm{mas} \mathrm{yr}^{-1}$ and

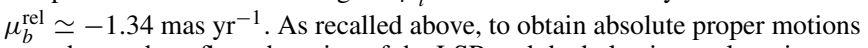
we subtract the reflected motion of the LSR and the bulge internal motion. Assuming $V_{\mathrm{LSR}}=-243 \mathrm{~km} \mathrm{~s}^{-1}$, at a distance of $8.4 \mathrm{kpc}$ from the Galactic center (these values are explained below) we obtain $\left(\mu_{l}, \mu_{b}\right)_{\mathrm{LSR}} \simeq(6.102,0) \mathrm{mas}_{\mathrm{yr}}-1$.
}

directed toward increasing $l$. After adding $v_{\mathrm{T}}$ to $V_{\mathrm{LSR}}$, the composite motion of the bulge becomes $\left(\mu_{l}, \mu_{b}\right)_{\text {bulge }+\mathrm{LSR}}=$ $(6.127,0) \mathrm{mas} \mathrm{yr}^{-1}$, and subtracting it from the relative motion quoted above for HP 1 yields $\mu_{l} \times \cos b=-0.65 \pm$ $0.39 \mathrm{mas} \mathrm{yr}^{-1}$ and $\mu_{b}=-1.34 \pm 0.39 \mathrm{mas} \mathrm{yr}^{-1}$. Using this proper motion, in the next section we calculate the cluster orbit.

\subsection{HP 1's Orbit in the Galaxy}

The orbit of HP 1 was computed both with the axisymmetric model by Allen \& Santillan (1991) and with a model including a bar. The models and integration algorithm are described by L. Jilkova et al. (2011, in preparation). Similar models were used in Magrini et al. (2010). Compared to these earlier versions, the axisymmetric model was rescaled to match the more recent values of rotation velocity, solar Galactocentric distance, and solar velocity relative to the LSR. Reid et al. (2009) estimated a rotation velocity of $254 \pm 16 \mathrm{~km} \mathrm{~s}^{-1}$ and a distance of $8.4 \pm 0.6 \mathrm{kpc}$, using the solar motion relative to the LSR determined by Dehnen \& Binney (1998). However, the analysis of Dehnen \& Binney was recently reexamined by Schönrich et al. (2010) who obtained slightly different values - the component in the direction of solar Galactic rotation was found to be $7 \mathrm{~km} \mathrm{~s}^{-1}$ higher. Taking this into account, we rescaled the Allen \& Santillan (1991) parameters to get values of $243 \mathrm{~km} \mathrm{~s}^{-1}$ at $8.4 \mathrm{kpc}$, which is also consistent with the results of Reid \& Brunthaler (2004), who obtained the solar rotation velocity from the proper motion of Sgr A*.

The Galactic bar is modeled by a Ferrers potential of an inhomogeneous triaxial ellipsoid (Pfenniger 1984). The model parameters are adopted from Pichardo et al. (2004) with a length of $3.14 \mathrm{kpc}$, an axis ratio of 10:3.75:2.56, a mass of $0.98 \times 10^{10} M_{\odot}$, an angular velocity of $60.0 \mathrm{~km} \mathrm{~s}^{-1} \mathrm{kpc}^{-1}$, and an initial angle with respect to the direction toward the Sun of $20^{\circ}$ (in the direction of Galactic rotation). For the axisymmetric background, we keep the potential described above with decreased bulge mass near the mass of the bar.

The initial conditions for the orbit calculations are obtained from the observational data characterizing the cluster: coordinates, distance to the Sun, radial velocity, and proper motion. To evaluate the impact of the measurement errors, we calculate a set of 1000 orbits with initial conditions given by sampling the distributions of observational inputs. We assume normal distributions for the distance to the Sun, radial velocity, and proper motion. The errors on radial velocity and proper motion components are given above, while for the distance to the Sun we assumed an error of $10 \%$.

The transformation of the observational data to the Cartesian coordinate system centered on the Sun was carried out with the Johnson \& Soderblom (1987) algorithm. The velocity vector with respect to the LSR is then obtained by correcting for the solar motion with respect to the LSR from Schönrich et al. (2010): $(U, V, W)_{\odot}=(11.1,12.24,7.25) \mathrm{km} \mathrm{s}^{-1}$ (right-handed system, with $U$ in the direction toward the Galactic center and $V$ in the Galactic rotation direction). The final transformation to the Galactocentric coordinate system was made by using a solar Galactocentric distance of $8.4 \mathrm{kpc}$ and a LSR rotation velocity of $243 \mathrm{~km} \mathrm{~s}^{-1}$ (see above). We obtain proper motions in equatorial coordinates $\mu_{\mathrm{RA}} \times \cos \mathrm{DEC}=0.76 \pm 0.39 \mathrm{mas} \mathrm{yr}^{-1}$ and $\mu_{\mathrm{DEC}}=-1.28 \pm 0.39 \mathrm{mas} \mathrm{yr}^{-1}$, and Cartesian coordinates and velocities $(x, y, z)=(1.59,0.31,0.25) \mathrm{kpc}$ and $\left(v_{x}, v_{y}, v_{z}\right)=(-57.47,-231.99,-34.26) \mathrm{km} \mathrm{s}^{-1}$. We adopted 

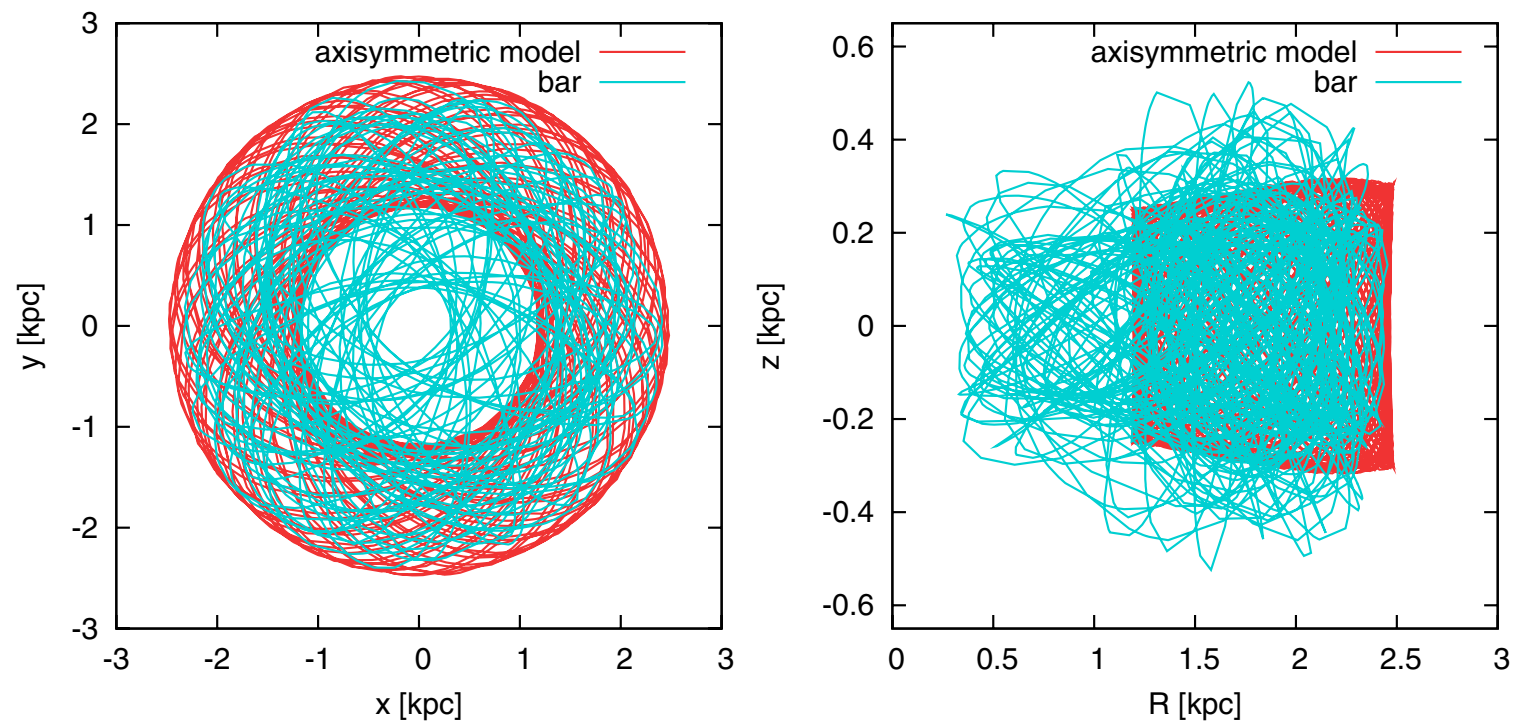

Figure 7. Galactic orbit of the cluster. Projections into the Galactic and meridional planes are plotted in the left and right panels, respectively. Orbit in the axisymmetric model is plotted by the red line and the orbit in the model including a bar by the blue line. Initial conditions are given by mean observational input data; see the text for a detailed description of integrations.

(A color version of this figure is available in the online journal.)
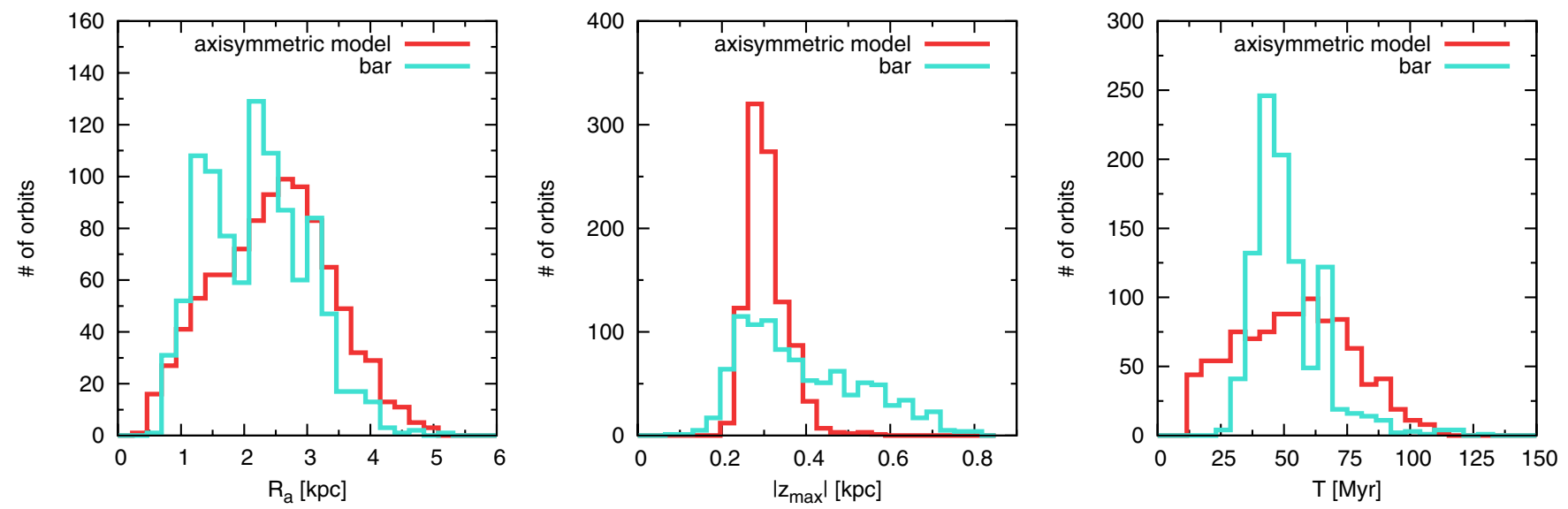

Figure 8. Distributions of orbital parameters—apogalacticon $R_{\mathrm{a}}$, vertical height of orbit $\left|z_{\max }\right|$, and phase period $T$. Distributions given by the axisymmetric model are plotted by the red line and in the model with a bar by the blue line (same as in Figure 7).

(A color version of this figure is available in the online journal.)

a right-handed, Galactocentric Cartesian system ( $x$ toward the Sun direction, $z$ toward the north Galactic pole).

We integrate the orbits with such initial conditions backward for an interval of $3 \mathrm{Gyr}$ using a Bulirsch-Stoer integrator with adaptive time step (Press et al. 1992). An example of orbits given by the average values of the observational data is given in Figure 7. The presence of the bar disturbs the orbit of this central globular cluster, causing deviations not found in the axisymmetric model. This can be considered as an upper limit for the excursions that the cluster can make inward and outward. Even so, it is clear that the cluster is essentially confined within the bulge.

Running simulations for a longer time is not very meaningful because there is evidence that the bar structure is a transient feature. For example, Minchev et al. (2010) suggest that the current bar might have formed only 2 Gyr ago. It is impossible to simulate the orbit along the entire life of the Milky Way, but one can guess that older bars would have had a similar effect on the orbit of HP 1 . Note that we also included the spiral arms, but they do not change the orbit significantly. They are very weak and the orbit is too close to the Galactic center to be influenced by any radial migration due to bar and spiral arm interaction.

We calculated orbital parameters as averaged values over individual revolutions in the Galactic plane for each orbit. Distributions for apogalacticon $R_{\mathrm{a}}$, vertical height of orbit $\left|z_{\max }\right|$, and phase period $T$ are shown in Figure 8. In general, the orbits do not reach galactic distances larger than $5 \mathrm{kpc}$ and the cluster remains close to the Galactic plane $\left(\left|z_{\max }\right|<0.3 \mathrm{kpc}\right.$ for the axisymmetric model, $\left|z_{\max }\right|<0.6 \mathrm{kpc}$ for the model including the bar).

For comparison purposes, we also computed the HP 1 orbit using the code developed by Mirabel et al. (2001) that includes the Galactic spheroidal and the disk potentials. It was recently applied to $\omega$ Centauri orbital simulations (Salerno et al. 2009). By this method we essentially use the same initial conditions $\left(U_{\circ}, V_{\circ}, W_{\circ}\right)$ as in the method described above, and the simulation results agreed well with the previous method for the barless model. 


\section{CONCLUSIONS}

The clear definition of an extended BHB morphology obtained from these high spatial resolution data, as provided using the proper motion cleaning method, indicates a very old age for HP 1 of $\sim 1$ Gyr older than the halo average.

The proper motions and orbits derived indicate that HP 1 does not wade into the halo and is confined within the Galactic bulge. As a result, HP 1 can be identified as a representative relic of an early generation of star clusters formed in the protoGalaxy. The very old globular cluster NGC 6522, also having moderate metallicity and a $\mathrm{BHB}$, is also confined within the bulge (Terndrup et al. 1998). Compared with the template metalrich bulge globular cluster NGC 6553 (Zoccali et al. 2001; Ortolani et al. 1995), HP 1 appears to have a more eccentric orbit, and it is much closer to the Galactic center.

Extensive tests of orbits within potential wells that include massive bars show that the confinement of HP 1 within the bulge is maintained even in the case of random orbits generated by the presence of the bar.

The case of HP 1, revealed using wide-field multi-conjugate adaptive optics, shows that such ground-based facilities can be used for high spatial resolution studies of crowded inner bulge clusters. Such data can provide a much better understanding of globular cluster subsystems, their connection with stellar populations in the Galaxy, and the sequence of processes involved in the formation of the Galaxy itself.

We are grateful to Ata Sarajedini for helpful discussions on the second parameter effect. We thank the referee for important suggestions on the proper motion analysis. We thank the ESO Adaptive Optics group, in particular, Enrico Marchetti and Paola Amico. B.B. and E.B. acknowledge grants from the Brazilian agencies CNPq and FAPESP. S.O. acknowledges the Italian Ministero de l'Università e della Ricerca Scientifica e Tecnologica.

\section{REFERENCES}

Allen, C., \& Santillan, A. 1991, RevMexAA, 22, 255

Anderson, J., Bedin, L. R., Piotto, G., Yadav, R. S., \& Bellini, A. 2006, A\&A, 454, 1029

Barbuy, B., Bica, E., \& Ortolani, S. 1998, A\&A, 333, 117

Barbuy, B., Zoccali, M., Ortolani, S., Hill, V., Minniti, D., Bica, E., Renzini, A., \& Gomez, A. 2009, A\&A, 507, 405

Barbuy, B., et al. 2006, A\&A, 449, 349

Bica, E. 1988, A\&A, 195, 76

Bono, G., et al. 2010, ApJ, 708, L74

Bouy, H., Kolb, J., Marchetti, E., Martín, E. L., Huélamo, N., \& Barrado Y Navascués, D. 2008, A\&A, 477, 681

Buonanno, R., Corsi, C. E., \& Fusi Pecci, F. 1989, A\&A, 216, 80

Campbell, M. A., Evans, C. J., Mackey, A. D., Gieles, M., Alves, J., Ascenso, J., Bastian, N., \& Longmore, A. J. 2010, MNRAS, 405, 421
Dehnen, W., \& Binney, J. J. 1998, MNRAS, 298, 387

Dotter, A., et al. 2010, AJ, 708, 698

Feltzing, S., \& Johnson, R. A. 2002, A\&A, 385, 67

Ferraro, F. R., et al. 2009, Nature, 462, 483

Gao, L., Theuns, T., Frenk, C. S., Jenkins, A., Helly, J. C., Navarro, J., Springel, V., \& White, S. D. M. 2010, MNRAS, 403, 1283

Gillessen, S., Eisenhauer, F., Trippe, S., Alexander, T., Genzel, R., Martins, F., \& Ott, T. 2009, ApJ, 692, 1075

Howard, C. D., Rich, R. M., Reitzel, D. B., Koch, A., De Propris, R., \& Zhao, H. 2008, ApJ, 688, 1060

Johnson, D. R. H., \& Soderblom, D. R. 1987, AJ, 93, 864

Kuijken, K., \& Rich, R. M. 2002, AJ, 124, 2054

Lee, Y.-W., Demarque, P., \& Zinn, R. 1994, ApJ, 423, 248

Lupton, R. H., \& Monger, P. 1997, The SM Reference Manual, http://www.astro.princeton.edu/ rhl/sm/sm.html

Magrini, L., Randich, S., Zoccali, M., Jilkova, L., Carraro, G., Galli, D., Maiorca, E., \& Busso, M. 2010, A\&A, 523, 11

Majaess, D. J., Turner, D. G., \& Lane, D. J. 2009, MNRAS, 398, 263

Marchetti, E., et al. 2007, Messenger, 129, 8

Marigo, P., Girardi, L., Bressan, A., Groenewegen, M. A. T., Silva, L., \& Granato, G. L. 2008, A\&A, 482, 883

Minchev, I., Boily, C., Siebert, A., \& Bienayme, O. 2010, MNRAS, 407, 2122

Mirabel, I. F., Dhawan, V., Mignani, R. P., Rodrigues, I., \& Guglielmetti, F. 2001, Nature, 413, 139

Momany, Y., Ortolani, S., Bonatto, C., Bica, E., \& Barbuy, B. 2008, MNRAS, 391,1650

Momany, Y., et al. 2003, A\&A, 402, 607

Nakasato, N., \& Nomoto, K. 2003, ApJ, 588, 842

Ortolani, S., Barbuy, B., \& Bica, E. 1995, Messenger, 82, 20

Ortolani, S., Bica, E., \& Barbuy, B. 1997, MNRAS, 284, 692

Ortolani, S., Renzini, A., Gilmozzi, R., Marconi, G., Barbuy, B., Bica, E., \& Rich, R. M. 1995, Nature, 377, 701

Pfenniger, D. 1984, A\&A, 134, 373

Pichardo, B., Martos, M., \& Moreno, E. 2004, ApJ, 609, 144

Press, W. H., Teukolsky, S. A., Vetterling, W. T., \& Flannery, B. P. 1992 Numerical Recipes (Cambridge: Cambridge Univ. Press)

Reid, M. J., \& Brunthaler, A. 2004, ApJ, 616, 872

Reid, M. J., et al. 2009, ApJ, 700, 137

Rey, S.-C., Yoon, S.-J., Lee, Y.-W., Chaboyer, B., \& Sarajedini, A. 2001, AJ, 122,3219

Rich, R. M. 1988, AJ, 95, 828

Salerno, G., Bica, E., Bonatto, C., \& Rodrigues, I. 2009, A\&A, 498, 419

Sana, H., Momany, Y., Gieles, M., Carraro, G., Beletsky, Y., Ivanov, V. D., de Silva, G., \& James, G. 2010, A\&A, 515, A26

Sandage, A., \& Wildey, R. 1967, ApJ, 150, 469

Schönrich, R., Binney, J., \& Dehnen, W. 2010, MNRAS, 403, 1829

Stetson, P. B. 1994, PASP, 106, 250

Terndrup, D. M., Popowski, P., Gould, A., Rich, R. M., \& Sadler, E. M. 1998, AJ, 115,1476

Terzan, A. 1964a, Haute Prov. Publ., 7, 2

Terzan, A. 1964b, Haute Prov. Publ., 7, 3

Terzan, A. 1965, Haute Prov. Publ., 8, 11

Terzan, A. 1966, Haute Prov. Publ., 8, 12

Tiede, G. P., \& Terndrup, D. M. 1999, AJ, 118, 895

Valenti, E., Ferraro, F. R., \& Origlia, L. 2004, MNRAS, 351, 1204

Vanhollebeke, E., Groenewegen, M. A. T., \& Girardi, L. 2009, A\&A, 498, 95

Zhao, H. 1996, MNRAS, 283, 149

Zoccali, M., Renzini, A., Ortolani, S., Bica, E., \& Barbuy, B. 2001, AJ, 121, 2638 